\title{
ORTHOSTATIC HYPOTENSION: UNCOMMON SIDE EFFECT OF ARIPIPRAZOLE
}

\author{
Kazuhito Takahashi ${ }^{1,2}$, Toshinori Nakamura ${ }^{1}$, Daimei Sasayama ${ }^{1} \&$ Shinsuke Washizuka ${ }^{2}$ \\ ${ }^{l}$ Department of Psychiatry, Shinshu University School of Medicine, Matsumoto, Nagano, Japan \\ ${ }^{2}$ Department of Psychiatry, Ritsuzankai Iida hospital, Iida, Nagano, Japan
}

received: 1.12.2020;

revised: 9.1.2021;

accepted: 17.1.2021

$* * * * *$

\section{INTRODUCTION}

Aripiprazole is a second-generation antipsychotic drug, which acts as a partial agonist of the dopamine D2 and serotonin $1 \mathrm{~A}$ receptors and as an antagonist of the serotonin 2A receptors. Orthostatic hypotension is a common side effect of prescription drugs. However, aripiprazole rarely induces orthostatic hypotension due to its low affinity for $\alpha 1$ adrenergic receptors.

The American College of Cardiology/American Heart Association/Heart Rhythm Society (ACC/AHA/HRS) guidelines recommend cessation of the offending drug and addition, if necessary, of midodrine hydrochloride or fludrocortisone in patients with drug-induced orthostatic hypotension (Shen et al. 2017). In the present case, orthostatic hypotension with syncope was suspected to be caused by aripiprazole. The symptoms disappeared after starting midodrine hydrochloride, and the dose of aripiprazole was gradually discontinued over 2 weeks after which midodrine was successfully discontinued without recurrence of hypotension.

\section{CASE REPORT}

A 30-year-old woman with a history of depressive episode, presented with a manic state and was admitted to our psychiatric ward. She did not have a history of any other significant medical problems, manic episodes, or a family history of mental illness. Her vital parameters, physical examination, and laboratory data revealed no significant abnormalities. The patient was diagnosed with bipolar I manic episode, according to the Diagnostic and Statistical Manual of Mental Disorders, Fifth Edition. On the day of hospitalization, aripiprazole $24 \mathrm{mg} / \mathrm{d}$ was started. On day 7, her manic symptoms, such as flight of ideas, excessive talkativeness, and elevated mood disappeared. However, the non-specific mental symptoms, such as insomnia, hyperacusis, and the feeling of being observed persisted. On day 7 , the dose of aripiprazole was reduced to $18 \mathrm{mg} / \mathrm{d}$ to avoid oversedation, and lamotrigine $25 \mathrm{mg} / \mathrm{d}$ was started to prevent relapse. On day 12 , she began to complain of dizziness and weakness, and these symptoms gradually deteriorated. Ramelteon $8 \mathrm{mg} / \mathrm{d}$ was started for insomnia, and brotizolam $0.25 \mathrm{mg} / \mathrm{d}$ and flunitrazepam $1 \mathrm{mg} / \mathrm{d}$ were used as needed. On day 14 , she began to fall repeatedly. The Schellong test showed that her sitting blood pressure and pulse rate were 118/83 $\mathrm{mmHg}$ and 97 beats/min, respectively. However, her blood pressure was undetectable and her pulse in the radial artery was barely detectable when standing. During the Schellong test, the patient had a syncope that caused her to fall backward. No seizures were observed, and her syncope lasted for about $30 \mathrm{~s}$. One minute after recovering from her syncope, her blood pressure improved to $139 / 96 \mathrm{mmHg}$, and pulse rate was 100 beats/min. Echocardiography and blood tests were immediately performed, but no abnormalities were found. Therefore, we suspected drug-induced orthostatic hypotension due to either lamotrigine or aripiprazole. As lamotrigine was her main medication to prevent relapse, we decided to first reduce the dose of aripiprazole to $12 \mathrm{mg} / \mathrm{d}$ and start midodrine hydrochloride at $4 \mathrm{mg} / \mathrm{d}$. Orthostatic hypotension improved the next day, and she no longer fell or experienced syncope. Aripiprazole was gradually reduced to $3 \mathrm{mg} / \mathrm{d}$, and on day 29 , midodrine hydrochloride was discontinued. On day 35, aripiprazole was discontinued. Although lamotrigine was increased to $200 \mathrm{mg} / \mathrm{d}$, her orthostatic hypotension did not relapse. On day 44, her feeling of being observed, hyperacusis, and insomnia disappeared, and she was discharged.

\section{DISCUSSION}

The present case has two important clinical implications. One is that orthostatic hypotension with syncope may occur as a side effect of aripiprazole. The other is that, by using midodrine hydrochloride, the causative drug could be gradually reduced instead of being suddenly discontinued. There was no recurrence of orthostatic hypotension after tapering and discontinuation of aripiprazole and midodrine hydrochloride. Lamotrigine was increased gradually during treatment, but no exacerbation of orthostatic hypotension was observed. As there were no other apparent medical conditions, including pregnancy, which could have caused the orthostatic hypotension, aripiprazole was likely the causative drug. 
The patient was prescribed lamotrigine, ramelteon, flunitrazepam, and brotizolam. CYP3A4 is the major metabolizing enzyme for aripiprazole, flunitrazepam, and brotizolam, and is also involved in the metabolism of ramelteon. Therefore, competitive inhibition may have reduced the clearance of aripiprazole, enhancing the side effects. Although the clearance of the sleep medications may also have been reduced, they were unlikely to be the culprit medications for two reasons. First, orthostatic hypotension is an uncommon side effect of the abovementioned three medications. Second, no excessive somnolence or muscle weakness was observed, suggesting that their effects were not enhanced.

This is the first reported case, to our knowledge, of orthostatic hypotension with syncope caused by aripiprazole. The administration of midodrine hydrochloride was effective from the day the medication was started. As sudden discontinuation of antipsychotics increases the risk of worsening psychotic symptoms and causes neuroleptic malignant syndrome (Amore \& Zazzeri 1995), gradual reduction of the dose is preferred. This case showed that immediate discontinuation of the causative drug of orthostatic hypotension is not always essential and can be gradually reduced.

Few studies have previously reported orthostatic hypotension during aripiprazole treatment. Kane et al. (Kane et al. 2002) reported that orthostatic hypotension occurred in $7 \%$ of patients treated with $30 \mathrm{mg} / \mathrm{d}$ of aripiprazole. Furthermore, Ishigooka et al. (Ishigooka et al. 2006) reported that the occurrence of hypotension was $1.25 \%$. However, these results do not reflect the true numbers of orthostatic hypotension directly caused by aripiprazole, because antiparkinsonian drugs, which could also cause that effect, were used to treat extrapyramidal symptoms in 10\% (Kane et al. 2002), and $35 \%$ (Ishigooka et al. 2006) of the patients in these studies.

Determining the causative drug is challenging when multiple drugs are prescribed, which is often the case in psychiatric treatment. Patients with mental illness often suffer from physical illness (Kondo et al. 2017), which can also cause orthostatic hypotension, making the exact cause even more difficult to ascertain.

As mentioned above, the competitive inhibition of CYP3A4 may have enhanced orthostatic hypotension in the present case. The absence of an enhancing effect of sleeping medications, led us to presume that aripiprazole was the causative drug. Further investigation is necessary to elucidate the possible involvement of aripiprazole in orthostatic hypotension.

\section{Acknowledgments: None.}

\section{Conflict of interest: None to declare.}

\section{Contribution of individual authors:}

Kazuhito Takahashi: Manuscript writing, literature search.

Nakamura Toshinori: Manuscript writing, supervising of the writing of the paper.

Sasayama Daimei: Manuscript writing, supervising of the writing of the paper.

Washizuka Shinsuke: Manuscript writing, supervising of the writing of the paper.

\section{References}

1. Amore M, Zazzeri N: Neuroleptic malignant syndrome after neuroleptic discontinuation. Prog. Neuro-Psychopharmacol. Biol Psychiatry 1995; 19:1323-1334

2. Ishigooka J, Miura S, Koyama T, Toru M, Murasaki M, Yagi $G$ et al.: Clinical evaluation of aripiprazole in schizophrenic patients - A comparative double-blind study with haloperidol. Jpn J Clin Psychopharmacol 2006; 9:295-329

3. Kane JM, Carson WH, Saha AR, McQuade RD, Ingenito $G G$, Zimbroff $D L$ et al.: Efficacy and safety of aripiprazole and haloperidol versus placebo in patients with schizophrenia and schizoaffective disorder. J Clin Psychiatry 2002; 63:763-771

4. Kondo S, Kumakura Y, Kanehara A, Nagato D, Ueda T, Matsuoka $T$ et al.: Premature deaths among individuals with severe mental illness after discharge from long-term hospitalisation in Japan: a naturalistic observation during a 24-year period. BJPsych open 2017; 3:193-195

5. Shen WK, Sheldon RS, Benditt DG, Cohen MI, Forman DE, Goldberger ZD et al: 2017 ACC/AHA/HRS Guideline for the Evaluation and Management of Patients With Syncope: A Report of the American College of Cardiology/ American Heart Association Task Force on Clinical Practice Guidelines and the Heart Rhythm Society. Circulation 2017; 136:e39-e110

\section{Correspondence:}

Kazuhito Takahashi, MD

Department of Psychiatry, Shinshu University School of Medicine

3-1-1, Asahi, Matsumoto, Nagano 390-8621, Japan

E-mail: take@shinshu-u.ac.jp 